\title{
Aprendizaje de las ciencias basado en proyectos: del contexto a la acción
}

\author{
Neus Sanmartí, Conxita Márquez \\ Departament de Didàctica de les Matemàtiques i les Ciències Experimentals. Facultat de \\ Ciències de l'Educació. Universitat Autònoma de Barcelona. Barcelona. España
}

[Recibido el 1 de abril de 2017, aceptado el 5 de mayo de 2017]

Actualmente, se están impulsando numerosas metodologías didácticas basadas en el trabajo por proyectos. En relación a ellas se identifican algunas características comunes, aunque en la práctica se conceptualizan y aplican de manera muy diversa. Las diferencias principales radican en cómo se entiende qué es un contexto idóneo y cómo se selecciona, en los objetivos de aprendizaje relacionados con los conocimientos conceptuales a construir, en la forma de concebir y aplicar un proceso de investigación y, también, en qué se entiende por actuar y cómo se promueve. En el artículo se reflexiona, en relación a estos aspectos, sobre las concepciones y prácticas desde el punto de vista de la competencia científica, y sobre lo que se sabe y sobre lo que aún son campos abiertos en didáctica de las ciencias.

Palabras clave: proyectos, competencia científica, contexto, acción, ideas clave.

\section{Projects based learning science: from context to action}

Nowadays, many didactic methodologies framed on Project Based Learning (PBL) are being promoted. In relation to them, some common characteristics are identified, although they are conceptualized and applied rather differently. The main differences lie in: (1) the understanding of suitable context and its selection, (2) the learning objectives related to the conceptual knowledge to be constructed, (3) the way of conceiving and applying a research process and (4) what is understood for acting and how it is promoted. Regarding these aspects, the article provides a reflection on not only the conceptions and practices from the scientific competence view, but also the knowledge about open fields for research in science education.

Key words: projects, scientific literacy, context, action, key ideas.

Para citar el artículo. Sanmartí, N. y Márquez, C. (2017). Aprendizaje de las ciencias basado en proyectos: del contexto a la acción. Ápice. Revista de Educación Científica, 1(1), 3-16. DOI: https://doi.org/10.17979/arec.2017.1.1.2020

Contacto.Neus.sanmarti@uab.cat Conxita.marquez@uab.cat 
Actualmente, en todo el mundo, se vuelve a hablar de un trabajo en el aula que parte de aplicar una metodología de aprendizaje basada en la realización de "proyectos", planteados desde puntos de vista muy diversos. En el campo de la enseñanza de las ciencias, esta metodología conecta con líneas de investigación como las de "CienciaTecnología-Sociedad", "Ciencia en contexto", "Temas socio-científicos (SSI)", "Educación ambiental" (y en general, en relación a las llamadas temáticas transversales), "Aprendizaje por indagación", "Aprendizaje basado en la modelización", "Ciencia, Tecnología, Ingeniería, Matemática (STEM)", entre otras, marcos en los que también confluyen distintas concepciones y prácticas.

La metodología de trabajo por "proyectos", que parten de aprender alrededor de temáticas complejas que tengan interés y sentido para el alumnado, tiene una larga historia de más de 100 años desde John Dewey, que habló de una enseñanza centrada en el aprendiz o activa y William H. Kilpatrick, que la sistematizó en 1918, pasando por Ovide Decroly, Celéstin Freinet y Lawrence Stenhouse, entre otros muchos. También confluyen con estos planteamientos propuestas como las de "Aprendizaje basado en problemas" o "Aprendizaje y servicio" y, más recientemente, en el marco del nuevo currículo de Finlandia, se habla de "Aprendizaje basado en fenómenos". Pero además se están planteando nuevas propuestas de formatos de aprendizaje como las relacionadas con la "Clase invertida", la "Gamificación", el aprendizaje en "ambientes", las llamadas "Rutinas y estrategias de pensamiento" de David Perkins, etc. Por tanto, nos encontramos ante un amplio abanico de propuestas metodológicas, cada una con objetivos específicos y fundamentaciones teóricas particulares dentro de un amplio marco común que tiene como finalidad replantear la actividad educativa.

Ante este nuevo panorama muchos se preguntan sobre su validez. Los estudios realizados hasta ahora muestran que cada "metodología" cumple con sus objetivos aunque no necesariamente con los de las otras propuestas. Estos resultados ya se dieron cuando se analizaron los de los distintos proyectos promovidos en los años 60-70 para la enseñanza de las ciencias. En ese periodo, también de mucho cambio en los contenidos y métodos de enseñanza de las ciencias, las investigaciones realizadas mostraron que los resultados dependían de los propósitos de los autores de cada proyecto (Pintó, 1983).

En el contexto actual hay datos que demuestran que los alumnos que aprenden en el marco de proyectos son más creativos, más autónomos, más capaces de trabajar en equipo y que aumenta la motivación, entre otras muchas variables (Vega, 2015), pero ¿también son más capaces de utilizar el conocimiento científico para fundamentar la toma de decisiones en nuevas situaciones (transferir)? Y son más autónomos pero ¿ponen en duda y reconstruyen sus ideas previas? Y son más capaces de trabajar cooperativamente con otros pero ¿toman decisiones para actuar de forma sostenible ambientalmente?

Actualmente aun es más difícil pensar en una posible validación de las propuestas ya que dependen mucho de los objetivos de los profesores que las ponen en práctica, y de la forma como se interrelacionan. Además, como ya decían Johsua y Dupin (1993), las leyes de la didáctica que se puedan enunciar se refieren más a las cosas que no pueden suceder (constricciones) que a las que deberían suceder (prescripciones). En el marco de la sociedad actual, está bien demostrado que los llamados métodos tradicionales de enseñanza no posibilitan el desarrollo de la competencia científica en la mayoría de la población. Especialmente entre el alumnado que se caracteriza por tener un estilo motivacional de tipo sociable o curioso (Martín-Díaz y Kempa, 1991), o el que proviene 
de ambientes socioculturales poco estimulantes. Por todo ello nos encontramos ante nuevos retos y preguntas de investigación.

\section{¿Qué se entiende por competencia y, en concreto, por competencia científica?}

Alrededor del término "competencia" hay muchas definiciones y concepciones, y su uso inicial proviene de la formación profesional, con una visión muy atomística. Pero cuando a finales del siglo pasado se debatió el concepto en el marco de la OCDE ya se vio la necesidad de conceptualizarla de forma más holística. Una de las definiciones más consensuada es la de: "Capacidad de responder a demandas complejas y realizar tareas diversas de forma adecuada. Supone una combinación de habilidades prácticas, conocimientos, motivación, valores éticos, actitudes, emociones y otros componentes sociales y de comportamiento que se movilizan conjuntamente para lograr una acción eficaz" (DeSeCo, 2002). En ella se destaca que se trata de una capacidad compleja, que incluye muchos saberes y el conocimiento entre ellos, y que se demuestra en la acción.

En la misma línea, la definición de competencia científica dada en el marco de PISA (OECD, 2000), indica que es "la capacidad de usar el conocimiento científico, identificar preguntas y extraer conclusiones basadas en pruebas, con el fin de comprender y ayudar a tomar decisiones sobre el medio natural y los cambios que son consecuencia de la actividad humana". En relación a ella se habla de tres dimensiones: 1) la relacionada con los "procesos científicos" como, por ejemplo, reconocer preguntas científicamente investigables, o deducir y evaluar conclusiones; 2) la relacionada con los "conceptos científicos" que agrupaba alrededor de trece grandes ideas; y 3) la referida a las "situaciones y áreas de aplicación" que agrupa alrededor de tres grandes campos salud, medioambiente y tecnología- y la acción se dice que puede ser a nivel personal, local, global o histórica. Como se puede comprobar, la definición y concreción destaca el "para qué" aprender ciencias para toda la población, y lo relaciona con la toma de decisiones para actuar en base a pruebas.

En el último informe PISA (OECD, 2016) la visión de competencia científica ha evolucionado en parte y se podría discutir si es más idónea o no. En este caso se define como "la habilidad para interactuar con cuestiones relacionadas con la ciencia y con las ideas de la ciencia, como un ciudadano reflexivo". El énfasis se pone en poder entender y participar en debates críticos sobre temas de ciencia y tecnología. Para ello, se concretan tres (sub)competencias: a) Explicar fenómenos científicamente; b) Evaluar y diseñar la investigación científica; y c) Interpretar datos y pruebas científicamente. Se insiste que todas estas competencias requieren de conocimientos científicos, y los clasifica entre conocimientos de hechos, conceptos, ideas, teorías, conocimientos procedimentales y conocimientos epistémicos. Precisamente la diferenciación entre estos dos últimos es una aportación interesante en esta nueva aproximación al concepto de competencia científica, ya que discrimina entre el conocimiento de métodos y estrategias para "hacer ciencia", del conocimiento "sobre qué entendemos por ciencia". Y también añade el campo de las actitudes (hacia la ciencia, científicas y hacia la aplicación social de la ciencia).

Además se considera que una persona competente científicamente debe ser capaz de activar estos saberes en una gama de contextos personales, locales y globales y reconocer que la ciencia, la tecnología y la investigación en este campo son un elemento esencial de la cultura contemporánea que enmarca gran parte de nuestro pensamiento.

Como señalan Yus, Fernández, Gallardo, Barquin, Sepúlveda y Servan (2013), hay una 
contradicción entre la noción de competencia (movilización de saberes diversos), y hablar de competencias diversas asociadas a los tradicionales campos de conocimiento y, más aún, hablar de diversas competencias asociadas a procesos de la ciencia en el marco de la competencia científica. Si la competencia requiere la movilización de saberes diversos, no tiene demasiado sentido hablar de tantas competencias y, de hecho, el mismo documento de DeSeCo (2002) define sólo 3 competencias clave que, además, las interrelaciona: 1) Usar herramientas de manera interactiva (ej. lenguaje, tecnología), 2) Interactuar en grupos heterogéneos y 3) Actuar de forma autónoma.

Este breve repaso a la concepción de competencia nos muestra que es un concepto en construcción, que evoluciona a partir de reflexiones teóricas, del análisis de las prácticas escolares y, también, de la evolución de la sociedad. Los alumnos actuales, los llamados nativos digitales, viven en un mundo diferente, con otras reglas y actitudes y con otras necesidades de formación, tanto en contenidos como en métodos. Es posible que dentro de unos años utilicemos otro concepto para hablar de los objetivos de la escuela, pero es evidente que no será una vuelta al pasado.

\section{La metodología de "proyectos" como marco para un aprendizaje competencial}

Cuando se habla de un "aprendizaje basado en proyectos", actualmente se está hablando de metodologías muy diversas, aunque todas tienen algunos rasgos comunes (Sanmartí, 2016; Thomas, 2000). Algunos de los más relevantes se relacionan con que:

a) Se parte del estudio de alguna situación o problema contextualizado.

b) Se "investiga" para dar respuesta a preguntas, dudas o retos, iniciales o que van surgiendo a lo largo de la realización del proyecto.

c) Se aprenden, a partir del contexto y en respuesta a preguntas, conocimientos clave y transferibles a la interpretación y actuación en otros contextos.

d) Se incluyen contenidos y evaluaciones auténticas, con objetivos didácticos específicos.

e) Se da a los alumnos la oportunidad de trabajar relativamente autónomamente por periodos de tiempo extensos.

f) El profesor facilita pero no dirige.

g) Se trabaja en grupos heterogéneos, y se promueve el aprendizaje cooperativo y la reflexión.

h) Se utilizan herramientas para aprender de manera interactiva, promoviendo el uso de tecnologías digitales (cognitivas).

i) Se finaliza con alguna acción en el entorno que planifican los propios estudiantes.

Como se puede comprobar las tres últimas coinciden con las competencias claves definidas en el marco del documento de DeSeCo (2002), aunque todas ellas se pueden conceptualizar y aplicar de forma muy diversa. Las diferencias principales radican en cómo se entiende qué es un contexto idóneo y cómo se selecciona, en los objetivos de aprendizaje que tienen relación con los conocimientos conceptuales a construir, en la forma de concebir y aplicar un proceso de investigación y, también, en qué se entiende por actuar y cómo se promueve. A continuación se analizan, en relación a estos aspectos, distintas concepciones y prácticas desde el punto de vista de la competencia científica.

\section{a) ¿Qué se entiende por contexto y cómo se selecciona?}

Cuando se habla de contexto se refiere al escenario que se escoge para generar en el 
alumnado la necesidad de entender alguno de los fenómenos que pasan o han pasado en el mundo. Su comprensión, a través de la construcción y uso de unas ideas científicas y sobre la ciencia, debería capacitar a los aprendices para actuar en el marco de una comunidad de práctica.

Se considera que su potencialidad radica en que posibilita (LIEC, 2016):

- Reconocer la utilidad del conocimiento aprendido (tanto científica como en relación a la acción).

- Construir conocimiento científico con sentido y transferible.

- Generar una actividad científica escolar (indagar, argumentar y modelizar).

- Estimular la necesidad de aprender y de seguir aprendiendo.

- Generar emociones positivas en el alumnado al descubrir retos que le llevan a formular preguntas estimulantes, implicarse en la búsqueda de soluciones, y experimentar la satisfacción de encontrarlas.

- Implicarse en acciones que repercuten en la comunidad (escolar, del entorno próximo o global).

En el marco de los proyectos C-T-S como el Salters ya se indicaba que "Las unidades del curso deben empezar con aspectos de la vida de los estudiantes, que ellos hayan experimentado personalmente o través de los medios, y los conceptos científicos deben irse introduciendo a medida que son necesarios" (Campbell y Lubben, 2000). Y en la misma línea, King (2012) propone que "Una metodología basada en contextos consiste en aplicar la ciencia a una situación del mundo real que se usa como estructura central para la enseñanza".

A menudo se discute si el contexto siempre tiene que relacionarse con el mundo del alumnado y generar acciones relevantes socialmente. Pero hay acuerdo en que un contexto histórico forma parte de su cultura y, según como se trabaje a partir de él, también puede ayudar a tomar decisiones de actuación en el presente. Por ejemplo, un proyecto sobre la Alquimia comportó profundizar en qué se entiende por ciencia y generar acciones para dar a conocer afirmaciones que se encuentran en Internet y que no se pueden considerar científicas.

La selección del contexto acostumbra a ser muy diferente en primaria y secundaria. En general, en primaria se considera que el tema lo han de escoger los alumnos, mientras que en secundaria lo seleccionan los profesores en función del conocimiento científico que se quiere ayudar a construir.

En un estudio realizado (Garriga, Pigrau y Sanmartí, 2012) se comprobó, en buena parte de los casos analizados, que la forma clásica de escoger temas o contextos en primaria no promueve que el alumnado se plantee preguntas que generen un aprendizaje de ideas científicas significativas, ni un proceso de construcción de dichas ideas que se fundamente en la indagación. Como ya hace años escribía Cousinet (1967), no debe confundirse la curiosidad que es algo que se satisface rápidamente, con el interés, que se mantiene en el tiempo y se relaciona con lo que hoy llamamos indagar. En ese estudio también se comprobó que las "buenas preguntas" se generan a lo largo de un proyecto, cuando los alumnos observan fenómenos o manipulan objetos, y no al inicio, que acostumbran a ser mucho más estereotipadas y orientadas a buscar información.

En general hay una cierta tensión entre el contexto escogido y las ideas científicas que el profesorado pretende que los estudiantes aprendan. Está tensión se suele resolver favoreciendo o las ideas del modelo teórico o las del contexto. 
En el primer caso, se trabajan el máximo de ideas del modelo aunque no todas ellas sean necesarias para resolver, comprender o actuar en relación a la situación en la que se contextualiza el aprendizaje. En consecuencia, muchas veces el contexto se convierte en el pretexto para enseñar los conocimientos de manera similar a un currículo tradicional (Kortland, 2007; Marchán-Carvajal y Sanmartí, 2015).

En el segundo caso, sólo se plantea el aprendizaje de aquello que se necesita para resolver, comprender o actuar en relación a la situación en la que se contextualiza el aprendizaje. Generalmente, el objetivo no es tanto construir ideas clave o modelizar, sino instrumentalizar o aplicar conocimientos ya aprendidos y profundizar en competencias más transversales. En este caso, puede suceder que el problema a resolver se centre excesivamente en el del contexto y que los alumnos acaban siendo "expertos" conocedores de informaciones acerca de él, pero que no aprendan un conocimiento que puedan aplicar para argumentar en relación a otros problemas similares, es decir, no se promueve que los nuevos aprendizajes sean transferibles (Sanmartí, Burgos y Nuño, 2011). Por ejemplo, los estudiantes pueden estar muy bien informados sobre la vida del Dragón de Komodo y conocer que es una especie vulnerable pero si no han modelizado qué necesita un ser vivo para sobrevivir en un medio, no podrán utilizar lo aprendido a argumentar sobre otras especies en peligro de extinción, y sobre como ser críticos con las informaciones que se encuentran.

De hecho se podría diferenciar, en un mismo marco de aprendizaje científico a partir del análisis de situaciones contextualizadas, entre dos tipos de finalidades. Por un lado las propuestas que inciden en el objetivo del aprendizaje de ideas de la ciencia, como es el caso de buena parte de los proyectos C-T-S, o de modelos teóricos a partir de aplicar procesos de indagación (Windschitl, Thompson y Braaten, 2008) y, por otro, las que tienen como finalidad la creación de algún producto nuevo o la realización de alguna acción, en las que los conocimientos científicos son un instrumento más entre otros, como es el caso de las propuestas relacionadas con STEM, SSI, entre otras muchas.

Posiblemente el futuro pasará por la combinación de los dos tipos de propuestas, la orientada a la construcción del modelo teórico y la orientada a su instrumentalización, a partir de combinar actividades que potencien las dos perspectivas (Domènech, 2017). Y ello tiene que ver con la discusión sobre finalidad de los aprendizajes en el marco escolar.

Por ejemplo, ante el objetivo de que los ciudadanos lleven a cabo actuaciones para el reciclaje de materiales, "la educación" que promueve un ayuntamiento será la de dar a conocer informaciones y normas. En cambio, la escuela es la institución donde los niños y jóvenes pueden aprender el conocimiento que proporciona razones para argumentar, de manera fundamentada, si es importante reciclar, si es una buena propuesta hacerlo tal como nos dicen o si habría otras maneras mejores de hacerlo. En este caso, el aprendizaje que se requiere es más costoso ya que conlleva cambiar concepciones previas, construir conocimientos complejos, apropiarse de las reglas de la argumentación para que esté fundamentada en el conocimiento y en pruebas (mucho más que en opiniones), atrapar nuevos valores, etc. $Y$ es más, lo que se aprende en la escuela debería ser útil también para actuar en nuevas situaciones y aprender a resolver problemas que aún no se han planteado.

En definitiva, se trata de buscar contextos que faciliten la conexión entre lo que se aprende en la escuela y lo que pasa en el mundo, de forma que el aprendizaje que se realice a partir de ellos capacite para actuar y para seguir aprendiendo. 


\section{b) La construcción de un conocimiento científico con sentido y transferible}

Las metodologías sobre cómo enseñar está cambiando de manera muy rápida pero parece que su finalidad sea enseñar lo mismo. $Y$ esto puede ser preocupante ya que ante una metodología por proyectos que requiere tiempo, es imprescindible elegir las ideas científicas que se quieren que los alumnos vayan construyendo por ellos mismos, interactuando con los compañeros y con los docentes a partir de actividades que les permitan expresar, evaluar-regular y usar sus ideas. Todo ello exige superar creencias muy enraizadas entre los docentes como la de que "si no lo explico antes no lo pueden entender", u otra que asimila aprender un conocimiento a recoger y repetir informaciones que se encuentran en los libros (y ahora en Internet).

Hay un cierto consenso en que no se trata de enseñar una gran cantidad de hechos o conceptos inconexos, sino unas grandes ideas (Harlen, 2010) o ideas clave (New Generation Science Standards, 2013) o modelos teóricos (Izquierdo, Espinet, García, Pujol y Sanmartí, 1999; Izquierdo y Adúriz, 2003). Los conocimientos a aprender habrán de ser pocos pero claves y generales, útiles para comprender y explicar muchos fenómenos de manera que sean transferibles, es decir, aplicables a la interpretación de muchos otros hechos y a la resolución de problemas no abordados específicamente en el marco del proyecto. Por tanto, en el marco de un proyecto no tendrá mucho sentido evaluar si los estudiantes conocen las características concretas del problema planteado, por ejemplo la falta de agua de un acuífero cercano al Instituto, sino si se han apropiado del concepto de balance en una cuenca hidrogeográfica y lo sepan aplicar al análisis de otras situaciones. Así, frente a la pregunta: "Por qué el agua se mueve más libremente a través de la arena que de la arcilla y que repercusiones tiene en los acuíferos", los estudiantes deberían ser capaces de identificar en qué tienen que pensar y qué pruebas han de obtener para darle respuesta. Ello comporta una manera de pensar el mundo en la que se pregunten sobre las características y estructura del terreno y del agua, las posibles dinámicas e interacciones $y$, finalmente, sean capaces de tomar una decisión sobre la ubicación de pozos o sobre cómo paliar la falta de agua en una zona.

Se trata de diferenciar, en palabras de Lamo de Espinosa (2004) entre información, conocimiento y sabiduría. La información está actualmente al alcance de todos y sólo nos permite responder a qué es y cómo es (un ser vivo, un hecho...), pero para comprenderla y analizarla críticamente es necesario disponer de conocimientos que posibiliten generar argumentos para decidir qué se puede hacer. Y la sabiduría es la que nos posibilita plantearnos qué debo hacer (actuar), e implica interrelacionar conocimiento y valores. Por tanto, si los estudiantes en el marco de un proyecto buscan respuestas a preguntas del tipo: ¿Qué es? ¿Cómo se llama? ¿Dónde se encuentra? ¿Qué come? ¿Qué clases hay?, sólo están aprendiendo a copiar informaciones. Pero en cambio, otras orientadas a identificar estructuras y regularidades (¿Qué tienen en común? ¿Cómo y por qué se interrelacionan las partes? ¿Cómo se explican las diferencias y las similitudes?), causas y consecuencias (¿Qué cambia y qué no cambia? ¿De qué depende el cambio? ¿Qué pasaría si no cambiara?), factores limitantes o favorecedores de cambios (¿Qué consecuencias conlleva...? ¿Cómo conseguir que un cambio sea sostenible ambientalmente?), se relacionan con la construcción de conocimiento y conllevan indagar (algo muy distinto de copiar).

Este conocimiento no se descubre "haciendo", ni por transmisión o repetición de informaciones. Hoy sabemos que aprendemos a partir de nuestras ideas previas, que casi siempre son alternativas a lo que las diversas ciencias han generado y actualmente están aceptadas. Por tanto, construir conocimiento comporta cambiar concepciones, y 
para ello no es suficiente ni "copiar" ni "hacer", ya que también es necesario interactuar con personas que aporten otras formas de mirar el hecho o fenómeno, y de pensar y hablar sobre él, por lo que la función del profesorado es clave. Las metodologías de aprendizaje pueden ser diversas pero todas ellas, para promover la construcción de conocimientos por parte de los estudiantes y hacerlo de manera que participen de la actividad a través de las prácticas científicas, deberían incidir en promover que los estudiantes investiguen, argumenten y evalúen sus ideas, y modelicen para generar explicaciones válidas (Osborne, 2014; Couso, 2014).

Paralelamente, la dificultad para la transferencia es uno de los principales problemas que ha de abordar la educación científica (Gilbert, Bulte y Pilot, 2011), especialmente en el marco de un aprendizaje basado en proyectos.

Centrar el aprendizaje en un determinado contexto puede conllevar que sólo se atribuya significado al conocimiento aprendido en su marco ya que estudios realizados muestran que la transferencia se favorece cuando se aprende en múltiples contextos (Bassok y Holyoak, 1989). Pero también es cierto que estos estudios no han abordado el cambio en el tipo de conocimientos a aprender, ni si esta capacidad de transferir está relacionada con la construcción de modelos teóricos o grandes ideas, ni de si se ha enseñado a los estudiantes a transferirlas. Ya hay estudios que demuestran, por ejemplo, que la metacognición aumenta la transferencia así como que es necesario abstraer modelos cuando se trabaja a partir de ejemplos concretos, o analizar los problemas o las tareas (el contexto) desde múltiples perspectivas (Solaz-Portalés y Sanjosé, 2007). Por tanto, es un campo de investigación didáctica abierto.

\section{c) La génesis de las preguntas y el planteamiento del proceso de indagación para darles respuesta}

Un contexto siempre es complejo y permite plantearse distintas preguntas. Cada una de ellas puede requerir profundizar en un modelo teórico diferente de la ciencia. Por ejemplo, si el contexto escogido es la medicalización de la sociedad y la pregunta que nos plateamos es "Todos los medicamentos curan pero ¿también tienen efectos secundarios?" se tratará de comprender cómo están interrelacionadas las distintas partes y funciones de nuestro cuerpo, desde una perspectiva sistémica, y así poder generar argumentos sobre conductas de medicalización que pueden suponer un riesgo para nuestra salud (Domènech, Márquez, Roca y Marbà, 2015). Pero si la pregunta que se plantea fuera "¿Es lo mismo un medicamento que una vacuna?", el conocimiento a construir se relacionaría con el sistema inmunitario. Y si fuera "¿Cómo se absorbe y se metaboliza un medicamento? se tendría que profundizar además en el modelo de cambio químico. Pero también puede suceder que la pregunta que nos planteemos no requiera construir un conocimiento científico sino aplicar una estrategia de recogida y análisis de datos, como sería en el caso de si la pregunta fuera "¿Qué pruebas tenemos de que la sociedad se está medicalizando en exceso?"

A veces se plantea una pregunta y se invita a los alumnos a investigar para responderla. Hay una cierta confusión entre preguntas investigables (aquellas que requieren pruebas para ser contestadas) y preguntas investigables científicamente (aquellas que requieren relacionar las pruebas con una idea científica clave) (Couso, 2014). Las que nos interesan son aquellas que su planteamiento este orientado desde la ciencia. Volviendo al ejemplo de la medicalizacion, saber cuántas personas están sobremedicadas no sirve para profundizar en ninguna idea científica a no ser que se tenga alguna teoría de la relación entre el exceso de medicalización y la manifestación de alguna patología. 
Formular una pregunta investigable científicamente (PIC) requiere aplicar también conocimientos sobre cómo se genera la ciencia y, en concreto, sobre qué es una variable y la distinción entre las que varían y las que se controlan en un experimento, y sobre cómo diseñar procesos para recoger datos. Pero en la formulación de una PIC la demanda puede ser sólo descriptiva si se concreta en ¿cuáles?, ¿cuántos?, ¿cómo es la relación?, ¿de qué depende qué?, en referencia a dos a más factores o variables que se pueden observar, medir y controlar. En cambio, en el marco de la concepción de competencia científica definida anteriormente, la pregunta debería ir más allá y preguntarnos ¿cómo es que depende de?, y para poder responderla será necesario construir ideas y modelos teóricos que no se pueden inducir de los experimentos pero que son los que los guían y les dan sentido.

Por todo ello, en el proceso de búsqueda de respuestas a preguntas, la investigación no debería limitarse a buscar información en internet y resumirla, ya que en este caso sólo se primaría la reproducción de conocimientos de contenidos conceptuales de ciencia (ideas atomísticas, leyes) y no implicaría cambios de fondo respecto a una metodología de aprendizaje tradicional. Pero tampoco el objetivo de la investigación que se pueda llevar a cabo en el marco de un proyecto debería ser sólo aprender "destrezas indagativas" o contenidos procedimentales, especialmente si son de bajo perfil cognitivo y que comunican una imagen de ciencia simplista. Seguir un camino lineal de observación, formulación de hipótesis, realización del experimento, obtención de datos y elaboración de conclusiones parece un camino muy científico, pero muestra una ciencia muy empirista y naive. Esta visión estaría en contraposición con las nuevas concepciones de la naturaleza de la ciencia que ponen de manifiesto la importancia de la cognición (el razonamiento), la comunicación (la argumentación) y la indagación (obtención de pruebas) en la actividad científica (Osbone, 2014; Garrido y Couso, 2016) sin olvidar la función que tiene el contexto social y las emociones en el desarrollo del conocimiento (Mellado, Borrachero, Brígido, Melo, Dávila, Cañada, Conde, Costillo, Cubero, Esteban, Martínez, Ruiz, Sánchez, Garritz, Mellado, Vázquez, Jiménez y Bermejo, 2014).

Sin subvalorar la importancia de que los alumnos aprendan procedimientos y técnicas, también deben desarrollar habilidades de razonamiento científico para plantearse buenas preguntas, analizarlas y darles respuestas, como son la comparación entre explicaciones, la búsqueda de pruebas que las avalen, la argumentación en base a ellas y a ideas teóricas, etc. Estas destrezas, al ser más difíciles de aprender deben trabajarse en cada proyecto ya desde la educación primaria (Metz, 2004).

Paralelamente, que los estudiantes (y los profesores) planteen preguntas investigables depende en buena parte de cómo se promueva el proceso de indagación en el aula y de su relación con la construcción de conocimientos científicos significativos (Furman, Barreto y Sanmartí, 2013). En este sentido, plantear el trabajo en el marco de un aprendizaje basado en proyectos como una investigación es una gran oportunidad para trabajar de manera interdependiente los conocimientos conceptuales, procedimentales y epistémicos necesarios para desarrollar la competencia científica.

\section{d) ¿Qué se entiende por capacitar para actuar eficazmente?}

Actualmente, en el marco de un aprendizaje competencial, la acción no es un añadido final, sino que da sentido al proyecto y posibilita dar respuesta a la famosa pregunta del alumnado de "para qué sirve lo que estamos aprendiendo". El propio título del proyecto puede llevar de manera intrínseca a la necesidad de una acción. Por ejemplo, en el 
proyecto: “¿Cómo eliminar las manchas?" niños y niñas de educación infantil se plantearon el problema de cómo limpiar las manchas de pintura de su bata. Tras un trabajo experimental en el que se contemplaron distintas variables (agua fría/caliente, con jabón/sin, etc.) construyeron explicaciones razonadas sobre cuál sería el mejor método. Pero al darse cuenta de la dificultad que comporta limpiar las manchas propusieron una actuación: intentar no mancharse tanto al pintar.

De hecho la acción da sentido a todo el proceso de aprendizaje. Al inicio será importante comprender la situación-problema y plantear unas primeras preguntas e ideas sobre aquello que se cree que es necesario aprender para poder llegar a realizar una acción con éxito. Mientras se van construyendo los conocimientos necesarios para fundamentar la toma de decisiones también se habrá de profundizar en los relacionados más específicamente con las posibles opciones de actuación. Y normalmente, en la etapa final es cuando se argumenta la opción escogida, se diseña la acción a emprender $y$, en algunos casos, se ejecuta y se evalúan sus resultados. Por tanto, no es un añadido final al proyecto, sino que lo impregna todo.

Llegar a ser competente para actuar democráticamente en función de conocimientos que se han generado a lo largo de la historia, es un aprendizaje complejo y que necesita tiempo y experiencia. Es mucho más que aprender a diseñar y aplicar propuestas a partir de opiniones personales o creencias. Implica también desarrollar capacidades relacionadas con el pensamiento crítico, la reflexión y la participación, que posibiliten incidir conscientemente en la toma de decisiones individuales y colectivas, teniendo como marco las reglas de juego de las sociedades democráticas. Suponen identificar los conflictos de valores que aparecen siempre que se han de tomar decisiones ante los problemas abiertos que plantea la vida, y aprender a ser consecuente ya que, por ejemplo, no tendría demasiado sentido reducir residuos en el ámbito escolar y no en el familiar, o ser capaz de argumentar la importancia del descanso y en cambio dormir pocas horas.

Tal como señalan Breinting y Mogensen (1999), la mayor dificultad reside en que el alumnado reconozca que es posible actuar e influir en los cambios $y$, consecuentemente, que tenga deseos e iniciativa para plantear propuestas y aplicarlas.

Está demostrado que muchos niños y jóvenes no pasan a la acción no tanto porque desconozcan cuáles son las maneras de actuar responsables e, incluso, cuáles son las razones que las justifican, sino porque creen que no es posible el cambio o que ellos no pueden hacer nada. En un estudio realizado por Cunil, Melià y Ferrés (1992), los alumnos de secundaria consideraban mayoritariamente que la especie humana se caracteriza por el individualismo y por la búsqueda del provecho personal, por lo que es imposible cambiar lo que ellos decían que era genético. Y también consideraban que el problema sólo lo podían resolver otros (políticos, empresarios...). En sus reflexiones decían que su acción no tendría ningún efecto, por lo que no valía la pena ni intentarlo. En otros tipos de proyectos, tipo STEM, en los que la acción se centra a menudo en diseñar artefactos, también se observa que muchos estudiantes no se creen capaces de imaginar propuestas, ni de inventar o crear. Esperan que otros las planteen y no confían en sus propias capacidades.

Para aumentar la confianza en que se puede influir en la solución de los problemas individuales y colectivos, se está de acuerdo en que es importante trabajar habilidades y estrategias que posibiliten la gestión de las emociones y la regulación de conflictos. Esta capacitación precisa de la existencia de grupos sociales que actúen, que encuentren 
placer en hacerlo y que no excluyan a nadie. Todo ello no es fácil en el contexto escolar actual y requiere que el colectivo de profesores de un centro también sea capaz de organizarse como un grupo social que coopera, y de encontrar placer en hacerlo sin excluir a nadie, ya que como señala Camps (1998) los valores se aprenden porque "se atrapan".

Se considera que los principales campos de actuación, en los que se necesita conocimiento científico para fundamentar las decisiones, se relacionan con la salud individual y colectiva, el uso sostenible de los recursos del medio, la calidad del medio ambiente, la prevención de riesgos y el diseño de proyectos que comporten un uso responsable de los avances científicos y de la tecnología (PISA-OECD, 2016). Son campos que se caracterizan por su relevancia social, incluso cuando se refieran a los más relacionados con proyectos tipo STEM, en los que los estudiantes también deberían tener en cuenta que sus propuestas sean sostenibles ambientalmente o que no incorporen riesgos que puedan perjudicar la salud de las personas.

Por tanto aprender a actuar comporta aprender a profundizar en lo que deberíamos hacer, por qué, en base a qué y cómo, y así llegar a adquirir algo de sabiduría.

\section{Reflexiones finales}

Las propuestas actuales relacionadas con el aprendizaje de las ciencias en contexto y en interrelación con conocimientos de otras áreas, no se pueden considerar meros cambios metodológicos. Implican cambios, entre otros aspectos, en la finalidad de su aprendizaje (para qué aprender ciencias), en la visión de qué se entiende por ciencia, y en la selección de los contenidos (qué conocimientos son los "potentes" y, por tanto, claves en un aprendizaje en el marco escolar). En una investigación reciente (Toro, 2016), se ha comprobado que cuando los profesores piensan en innovar (en ese caso en la universidad), se centran mucho en cambiar formas de trabajo (trabajo cooperativo, uso de nuevas tecnologías...) y, en cambio, no ponen en duda que se tenga que enseñar lo mismo y desde la misma concepción de ciencia.

El camino abierto promueve que nos planteemos muchas nuevas preguntas, que exigirán investigar e innovar, y también compartir propuestas, resultados, acciones... En este sentido, nos podemos felicitar de que tengamos una nueva revista que nos posibilite la comunicación entre los miembros de la comunidad que tiene como objetivo la mejora en la enseñanza de las ciencias.

\section{Agradecimientos}

Grupo LIEC (2009SGR1543 por AGAUR) y al proyecto financiada por la Dirección General de Investigación, Ministerio de Educación y Ciencia (EDU2015-66643-C2-1-P)

\section{Referencias bibliográficas}

Bassok, M. y Holyoak, K.J. (1989). Interdomain transfer between isomorphic topics in algebra and physics. Journal of Experimental Psychology: Learning, memory and cognition, 1, 153-166.

Breiting, S. y Mogensen, F. (1999). Action competence and environmental education. Cambridge Journal of Education, 29(3), 349-353.

Campbell, B. y Lubben, F. (2000). Learning science through contexts: Helping pupils make sense of everyday situations. International Journal of Science Education, 
22(3), 239-252.

Camps, V. (1998). La escuela ante el reto del saber práctico. Infancia y Aprendizaje, 82, 65-73.

Cousinet, R. (1967). La Escuela Nueva. Barcelona: Ed. Luis Miracle.

Couso, D. (2014). De la moda de "aprender indagando" a la indagación para modelizar: una reflexión crítica. XXVI Encuentro de Didáctica de las Ciencias Experimentales. Huelva (Andalucía). Recuperado de: http://uhu.es/26edce/actas/ docs/conferencias/pdf/26ENCUENTRO_DCE-ConferenciaPlenarialnaugural.pdf

Cunill, M.A., Melià, R. y Ferrés, C. (1992). Avaluació d'una campanya d'EA duta a terme a I'IFP 'La Garrotxa' d'Olot. Trabajo Master. Dep. Did. de las CC.EE. y de la Matemàtica de la UAB. Documento no publicado.

DeSeCo - OCDE. (2002). Definition and Selection of Competencies: Theoretical and Conceptual Foundations. Summary of the final report "Key Competencies for a Successful Life and a Well-Functioning Society". Recuperado de: http://www.portalstat. admin.ch/deseco/deseco_finalreport_summary.pdf

Domènech, A., Márquez, C., Roca, M. y Marbà Tallada, A. (2015). La medicalización de la sociedad, un contexto para promover el desarrollo y uso de conocimientos científicos sobre el cuerpo humano. Enseñanza de Las Ciencias, 33(1), 101-125.

Domènech, J. (2017). Treball per projectes i competència científica: una proposta metodològica per al disseny de Projectes d'Indagació. Perspectiva Escolar, en prensa.

Furman, M., Barreto Pérez, M. C. y Sanmartí, N. (2013). El proceso de aprender a plantear preguntas investigables. Educació Química, 14, 1-16.

Garrido, A. y Couso, D. (2016). Pre-service teachers' perceptions about modelling: first steps towards a reflective participation in scientific practices. En: J. Lavonen, $\mathrm{K}$. Juuti, J. Lampiselkä, A. Uitto y K. Hahl (Eds.), Electronic Proceedings of the ESERA 2015 Conference. Science education research: Engaging learners for a sustainable future, Part 13: Pre-service science teacher education (Co-ed. Evagorou, M. y Michelini, M.). Helsinki, Finland: University of Helsinki.

Garriga, N., Pigrau, T. y Sanmartí, N. (2012). Cap a una pràctica de projectes orientats a la modelització. Ciències, 21, 18-28.

Gilbert, J. K., Bulte, A. M. W. y Pilot, A. (2011). Concept development and transfer in Context-Based science education. International Journal of Science Education, 33(6), 817-837.

Harlen, W. (2010). Principios y grandes ideas de la educación en ciencias. Publicado por la Association for Science Education.

Izquierdo, M. y Adúriz-Bravo, A. (2003). Epistemological foundations of school science. Science \& Education, 12(1), 27-43.

Izquierdo, M., Espinet, M., García, M.P., Pujol, R.M. y Sanmartí, N. (1999). Caracterización y fundamentación de la ciencia escolar. Enseñanza de las Ciencias. Vol. № extra, 79-91.

Johsua, S. y Dupin, J.J. (1993). Introduction à la didactique des sciences et des mathématiques. Paris: PUF. 
King, D. (2012). New perspectives on context-based chemistry education: Using a dialectical sociocultural approach to view teaching and learning. Studies in Science Education, 48(1), 51-87.

Kortland, J. (2007). Context-based science curricula: Exploring the didactical friction between context and science content. In Paper presented at the ESERA 2007. Conference Malmö, Sweden.

Lamo de Espinosa, E. (2004). Información, Ciencia, Sabiduría. Fòrum de les cultures Barcelona 2004. Recuperado de: http://www.barcelona2004.org/www. barcelona2004.org/esp/banco_del_conocimiento/docs/PO_1_ES_LAMO.pdf

LIEC (2016). Ciències en context. Barcelona. Cienciesencontext. Recuperado de : http:// www.cienciesencontext.com/

Marchán-Carvajal, I. y Sanmartí, N. (2015). Criterios para el diseño de unidades didácticas contextualizadas: Aplicación al aprendizaje de un modelo teórico para la estructura atómica. Educación Química, 26(4), 267-274.

Martin-Diaz, M. J. y Kempa, R. F. (1991). Los alumnos prefieren diferentes estrategias didácticas de la enseñanza de las ciencias en función de sus caracteristicas motivacionales. Enseñanza de Las Ciencias, 9(1), 59-68.

Mellado, V., Borrachero, A.B., Brígido, M., Melo, L.V., Dávila, M.A., Cañada, F., Conde, M.C., Costillo, E., Cubero, J., Esteban, R., Martínez, G., Ruiz, C., Sánchez, J., Garritz, A., Mellado, L., Vázquez, B., Jiménez, R. y Bermejo, M.L. (2014). Las emociones en la enseñanza de las ciencias. Enseñanza de las Ciencias, 32(3), 11-36

Metz, K.E. (2004). Children's understanding of scientific inquiry: Their conceptualization of uncertainty in investigations of their own design. Cognition and Instruction, 22(2), 219-290.

New Generation Science Standards (2013). Recuperado de: http://www.nextgen science.org/

OECD (2000) Measuring student knowledge and skills: The PISA assessment of reading, mathematical and scientific literacy. Paris: OECD.

OECD (2016), PISA 2015 Assessment and Analytical Framework: Science, Reading, Mathematic and Financial Literacy. PISA, OECD Publishing, Paris.

Osborne, J. (2014). Teaching scientific Practices: Meeting the Challenge of Change. Journal of Science Teacher Education, 25(2), 177-196.

Pintó, R. (1983). Anàlisi de programes de Física per a l'ensenyament elemental. Publicacions de la Universitat Autònoma de Barcelona.

Sanmartí, N. (2016). Trabajo por proyectos: ¿Filosofía o metodología? Cuadernos de Pedagogia, 472, 1-6.

Sanmartí, N.; Burgos, B. y Nuño, T. (2011). ¿Por qué el alumnado tiene dificultad para utilizar sus conocimientos científicos escolares en situaciones cotidianas? Alambique: Didáctica de las Ciencias Experimentales, 67, 62-69.

Solaz-Portalés, J. y Sanjosé, V. (2007). Cognitive variables in science problem solving: A review of research. Journal of Physics Teacher Education on line, 4(2), 25-32.

Thomas, J. W. (2000). A review of research on project-based learning. The Autodesk 
Foundation, California.

Toro, G. P. (2016). Enseñanza en Educación Superior: Una aproximación a la evolución de la innovación en la enseñanza de la ciencia con uso de entornos tecnológicos. Tesis doctoral. UAB. Recuperado de: http://www.tesisenred.net/bitstream/ handle/10803/400153/gpt1de1. pdf?sequence=1\&isAllowed=y

Vega, V. (2015). Project-Based Learning Research Review: Evidence-Based Components of Success. Recuperado de: https://www.edutopia.org/pbl-research-evidencebased-components

Windschitl, M., Thompson, J. y Braaten, M. (2008). Beyond the scientific method: Model-based inquiry as a new paradigm of preference for school science investigations. Science Education, 92(5), 941-967.

Yus, R., Fernández, M., Gallardo, M., Barquín, J., Sepúlveda M.P. y Serván, M.J. (2013). Revista de Educación, 360, 557-576. 